\title{
Diffuse Lamellar Keratitis After Laser In Situ Keratomileusis
}

\section{Jayalekshmi Nithin* and Anwar}

Research Ophthalmology, Eye Care and Research, United Arab Emirates

*Corresponding Author: Jayalekshmi Nithin, Research Ophthalmology, Eye Care and Research, United Arab Emirates.
Received: May 29, 2021

Published: June 30, 2021

(C) All rights are reserved by Jayalekshmi

Nithin and Anwar.

\begin{abstract}
To examine the incidence of diffuse lamellar keratitis after LASIK. We report 2 cases of DLK appearing after the immediate postoperative period of LASIK. We have observed that the astigmatic shift may be assorted with the occurrence of this DLK. Our 2 cases of DLK was following myopic Lasik. The sterile infiltrates resolved over a period of 4-8 weeks on topical steroid therapy.
\end{abstract}

Keywords: Diffuse Lamellar Keratitis (DLK); Laser In Situ Keratomileusis (LASIK); Sands of the Sahara Syndrome

Diffuse lamellar keratitis (DLK) is a non-infectious inflammatory complication associated with laser in situ keratomileusis (LASIK). Post-LASIK sterile interface keratitis has also been described as "sands of the Sahara syndrome" and central focal interface opacity after LASIK.

The corneal infiltrates may be focal or multifocal but remain confined to the lamellar interface without extension, anterior chamber reaction, or associated epithelial defect. Common to all previously reported cases is an onset within 1 month after LASIK treatment, enhancement, or flap manipulation.

\section{Case Reports}

Case 1

A 30-year-old man who presented to our clinic for an evaluation for laser vision correction (LVC). He was in excellent general health and had no general or ocular contraindications to LVC. Corneal topography and pachymetry readings were within the normal range. His pre-operative prescription was $-3.75 \mathrm{Sph} 20 / 20$ OD and -3.25 Sph /-0.50 cyl x 80 20/20 OS.
He underwent bilateral sequential LASIK in January 2020, both procedures were without intraoperative complications, and he was sent home with our standard postoperative regimen of TobraDex (tobramycin dexamethasone, Alcon) and Vigamox five times daily with Hypotears gel @ bedtime and aggressive lubrication.

At the 1-day postoperative visit, he demonstrated UAVA of $20 / 20$ (B) OD and OS with auto refraction-1.00/-0.25 x 104(OD) and $-0.50 /-0.75 \mathrm{X} 135$ (OS) with remarkable clinical findings. SlitLamp Bio microscopy revealed the presence of mild DLK phenomenon. It involved a sector of the flap interface radiating from the superior temporal margin of the flap extending into the visual axis. Treatment was initiated with TobraDex every 2 hours for 3 days.

On day 6 he complaining of decreased unaided vision OU (OD > OS) and was seen the following day, at that time his UAVA decreased by 20/60 (OD) 20/40 (OS) with mild light sensitivity.

Slit lamp evaluation revealed a symmetrical, diffuse "haze" at the level of the interface in each eye. The rest of the ocular examina- 
tion was unremarkable. The patient stated that he had been using the postoperative medications as directed.

We discontinued the TobraDex and started prednisolone acetate every 2 hours. Over the next several visits the BCVA fluctuated and advised to continue FML forte four times daily.

His BCVA did not start to improve until 4 weeks postoperatively. At that time, he began to demonstrate a slow, steady resolution of haze with corresponding improvement in his vision. At the 6 month visit, his BCVA back to 20/25 OD and OS, with mild residual of hyperopic and astigmatism OU.

\section{Case 2}

A 25-year old women, who was seen in December 2019 for corneal assessment before laser refractive surgery with good general and ocular health for LVC, Corneal topography and pachymetry readings were within the normal range. She underwent bilateral LASIK in January 2020 and her pre-operative correction was -3.25 SPH 20/20 OD and -3.50 SPH 20/20 OS, without any intraoperative complications, discharged with our standard postoperative regimen (case 1).

The day after surgery, clinical examination shows diffuse granular infiltrates were visible in the flap interface without epithelial defects, particularly in Right eye and the suspicion of DLK was confirmed, UAVA recorded 20/30 OD and 20/25 OS with auto refraction -0.25/-0.75 x 87 (OD) and +0.25/-0.50 X 101 (OS), Advised to continue as same postoperative regimen.

Follow-up examination day 6 after surgery, she complained decreased vision OU, examination was remarkable for UCVA 20/30 (OU) and slitlamp biomicroscopy revealed a healing erosion with mild diffuse granular infiltrates with TobraDex (tobramycin dexamethasone, Alcon) and Vigamox five times daily. Her conditions began to improve in day 12 after treatment, and regimen was switched to prednisolone acetate every 3 hours and Vigamox five times daily.

At one month visit, her unaided vision improved to $20 / 20$ OU without any significant complaints, the keratitis completely resolved with mild residual of astigmatism.

\section{Discussion and Conclusion}

The cause of DLK not clearly defined. A rare postoperative complication of LASIK, non-specific diffuse interface keratitis. DLK has reported 24 - 48 hours post-operative period. Clinically DLK presents with varying severity and the patients symptoms recorded as decreased in vision and mild light sensitivity, the patients are generally asymptomatic, the haze is at the level of interface and tend to be seen diffusely throughout the flap area. Day one visual acuity is usually seems good, when the visual axis is involved the loss visual acuity has been reported to be severe as 20/60. Our case 1 had documented the flap interface radiating from the superior temporal margin of the flap extending into the visual axis bilaterally with fluctuated BCVA, whereas the clinical appearance resolved after 6 months postoperatively. Case 2 had diffuse granular infiltrates were visible in the flap interface with rapid recovery appeared in 4 week post-operative period. We propose the mechanism of intensive topical application of Prednisolone acetate and Vigamox.

These 2 cases to bring attention to the possibility of DLK occurring beyond the immediate postoperative period. Care must be taken to exclude infectious keratitis. However, the prognosis for recovery is good following intensive topical steroid treatment [1-3].

\section{Acknowledgement}

Supported in part by Laser Eye Care and Research Center, Dubai Health Care City.

\section{Bibliography}

1. Anne Chang-Godinich., et al. "Late Occurrence of Diffuse Lamellar Keratitis after Laser In Situ Keratomileusis”. Archives of Ophthalmology 119.7 (2001): 1074-1076.

2. Jimmy Jackson. Diffuse lamellar keratitis: early diagnosis, aggressive treatment key.

3. Joseph P Shovlin. DLK Adds insult to Injury -Review Of Optometry.

\section{Volume 4 Issue 7 July 2021 (c) All rights are reserved by Jayalekshmi Nithin and} Anwar. 